Vietnam Journal of Mechanics, VAST, Vol. 28, No. 2 (2006), pp. 67-73

\title{
VAN DER POL SYSTEMS WITH VARIOUS RESTORING ELEMENTS
}

\author{
NGuyen Van Dinh and Tran Kim Chi \\ Institute of Mechanics, VAST
}

\begin{abstract}
Van der Pol oscillators with various linear and cubic restoring elements are examined. The evolution of stationary self-excited oscillations is described and the role of self-excitation as that of restoring elements is shown. Numerical method on computer is used.
\end{abstract}

\section{INTRODUCTION}

The classical Van der Pol oscillator with linear restoring element has been presented in every book on nonlinear oscillations $[1,2]$. It is known that, when the self-excitation is absent, closed orbits are observed in the phase plane (free oscillation) encircling a stable center $O$ (stable equilibrium state). With the presence of self-excitation, $O$ becomes an unstable focus. A unique closed orbit $S$ (stationary self-excited oscillation) exists and all other orbits are spirals, asymptotically approaching $S$.

The situation becomes complicated with the presence of nonlinear restoring elements, especially if linear and nonlinear elements are of different (attracting, pushing) type. The aforesaid stable center $O$ can be transformed into saddle. Other critical points (centers, foci, saddles) together with critical orbits (homo - heteroclinic) can appear; closed orbits (stationary self-excited oscillations) can not exist or a great number of them can appear simultaneously.

Below, in order to obtain useful informations, some Van der Pol systems with various linear and cubic restoring elements will be considered. The evolution and qualitative characteristics of closed orbits (stationary self-excited oscillations) are of special interest. The numerical method on computer is used. Some results, especially the reestablishment of homo-heteroclinic orbits, can be proved by the Melnikov criterion.

\section{VAN DER POL SYSTEMS WITH LINEAR AND CUBIC ATTRACTING ELEMENTS}

The simplest case is that of Van der Pol systems whose linear and cubic restoring elements are of the same attracting type so that the differential equation can be written as:

$$
\ddot{x}+x=-\beta x^{3}+h\left(1-\alpha x^{2}\right) \dot{x} ; \quad \beta>0,
$$

where $x$ is an oscillatory variable; overdots denote differentiation with respect to time $t$; $x$ (in the left hand side) represents the linear attracting element and $-\beta x^{3}$ (in the right hand side) - the cubic attracting one; $h>0$ is coefficient of linear negative friction and $\alpha>0$ is that characterizing intensity of nonlinear positive friction. 
When $h=0$, one has a conservative system. Its phase portrait is full of closed orbits (periodic free oscillations) encircling the origin $O$ which is a stable center (stable equilibrium state).

With the presence of the self-excitation $h\left(1-\alpha x^{2}\right) \dot{x}$, a unique stationary self-excited oscillation will be established; it corresponds to a closed orbit $S$, encircling $O$ which becomes now an unstable focus; other orbits are spirals, asymptotically approaching $\mathrm{S}$. Increasing $\alpha, S$ is expanded. For the same values $\beta=1, h=0.5$, Figs. $1,2,3$, respectively correspond to $\alpha=0.5,1.0,1.5$. Qualitatively, the system under consideration is "similar" to the classical Van der Pol one. The difference consists in the "oscillation level" including the number and the intensity of high harmonics.

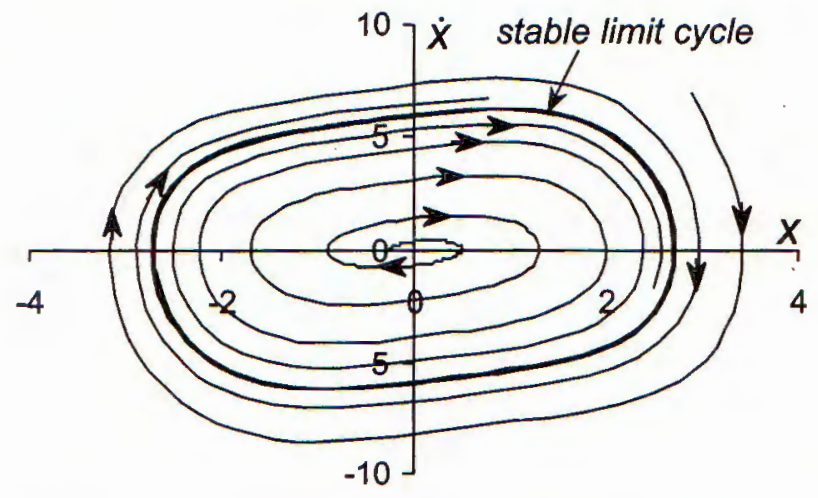

Fig. 1. The phase trajectories in the case $\beta=1, h=0.1, \alpha=0.5$

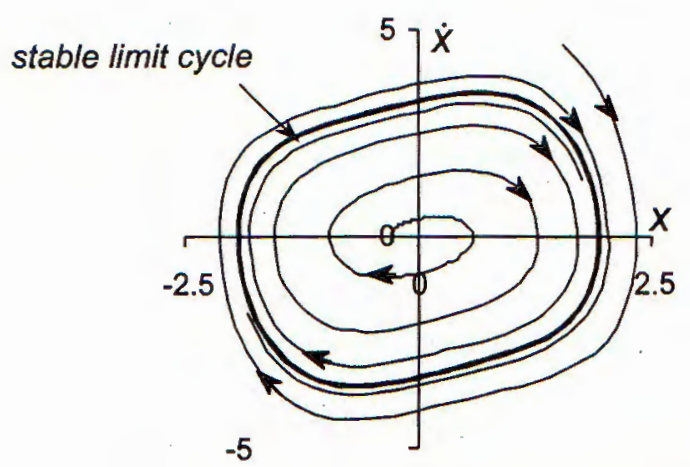

Fig. 2. The phase trajectories in the case $\beta=1, h=0.1, \alpha=1$

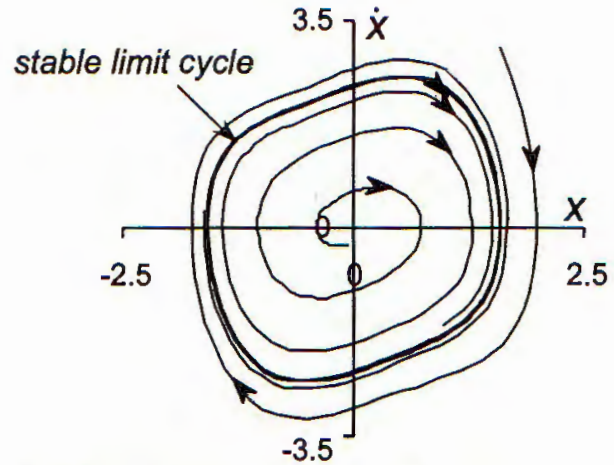

Fig. 3. The phase trajectories in the $\beta=1, h=0.1, \alpha=1.5$

\section{VAN DER POL SYSTEMS WITH LINEAR ATTRACTING AND CUBIC PUSHING ELEMENTS}

In this second example, linear and restoring elements belong to different types: the linear is attracting while the cubic is pushing. The differential equation now becomes:

$$
\ddot{x}+x=\beta x^{3}+h\left(1-\alpha x^{2}\right) \dot{x} ; \quad \beta>0 .
$$

In the phase plane of the originnal system $(h=0)$, there are three critical points: 
a stable center $O$ at the origin and two saddles at $I\left(-\frac{1}{\sqrt{\beta}}, 0\right)$ and $J\left(\frac{1}{\sqrt{\beta}}, 0\right)$ and six critical orbits. Among these orbits two most important ones are the upper and lower heteroclinic half-orbits $I J$ and $J I$. Closed orbits representing periodic free oscillations lie in the restricted domain bounded by two mentioned heteroclinic orbits (see Fig. 4 drawn with $\beta=1$ ).

In the presence of self-excitation, the heteroclinic orbit is destroyed and is replaced by four invariant manifolds, except at a particular value $\alpha=\alpha_{*}$ for which a new heteroclinic orbit is "reestablished". No closed orbit (self-excited oscillation) exists if $\alpha \leq \alpha_{*}$. Closed orbit appears only if $\alpha>\alpha_{*}$ and is subjected to contraction as $\alpha$ increases.

For the same values $\beta=1, h=0.1$, Figs. 5, 6, 7 correspond to $\alpha=1.0, \alpha_{*}=4.992498$, $\alpha=10.0$, respectively.

Using the Melnikov method $[3,4,5]$, the critical value $\alpha_{*}$ can be approximately determined. Choosing $\beta=1$, the unperturbed system $(h=0)$ is Hamiltonian. The Hamiltonian is $H(x, \dot{x})=\frac{x^{2}}{2}-\frac{x^{4}}{4}+\frac{\dot{x}^{2}}{2}$; the two saddles are $I(-1,0), J(1,0)$; the equation of the heteroclinic orbit is $H(x, \dot{x})=\frac{1}{4}$ or $\dot{x}= \pm \sqrt{2} \frac{1-x^{2}}{2}$, where $(+)$ corresponds to the upper half orbit $I J,(-)$ corresponds to the lower one $J I$. Applying to $I J$, the Melnikov function has an expression

$$
M=\int_{-\infty}^{+\infty} h\left(1-\alpha x^{2}(t)\right) \dot{x}^{2}(t) d t
$$

where $x(t)=\tanh \left(\frac{\sqrt{2}}{2} t\right)$ is a chosen motion law along $I J$ satisfying the initial conditions $x(0)=0, \dot{x}(0)=\frac{\sqrt{2}}{2}$.

After necessary calculations, the value of the Melnikov function is

$$
M=\frac{2 h \sqrt{2}}{15}(5-\alpha)
$$

Using the expression $\dot{x}=+\frac{\sqrt{2}\left(1-x^{2}\right)}{2}$ of $I J$, the same result is obtained

$$
\begin{aligned}
M & =\int_{-\infty}^{+\infty} h\left(1-\alpha x^{2}(t)\right) \dot{x}^{2}(t) d t=2 h \int_{0}^{\infty}\left(1-\alpha x^{2}(t)\right) \dot{x}^{2}(t) d t \\
& =h \sqrt{2} \int_{0}^{1}\left(1-\alpha x^{2}\right)\left(1-x^{2}\right) d x=\frac{2 h \sqrt{2}}{15}(5-\alpha) .
\end{aligned}
$$

Vanishing $M=0$, the critical value is found: $\alpha_{*}=5$. This value is close to that obtained above for $h=0.1$. When $h$ is increased, the accuracy is decreased, for example, $\alpha_{*}=4.859715$ for $h=0.5 ; \alpha_{*}=4.73617302$ for $h=0.8$. 


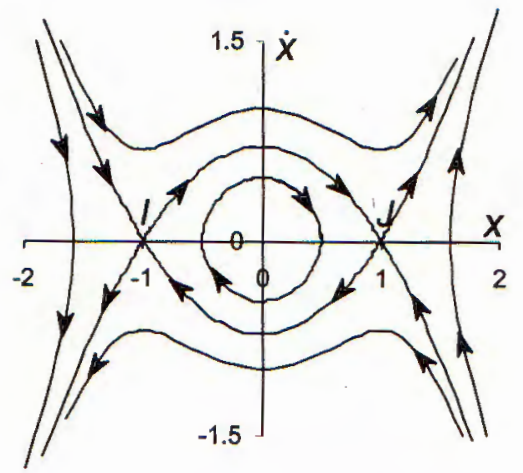

Fig. 4. The phase trajectories in the case

$$
\beta=1, h=0
$$

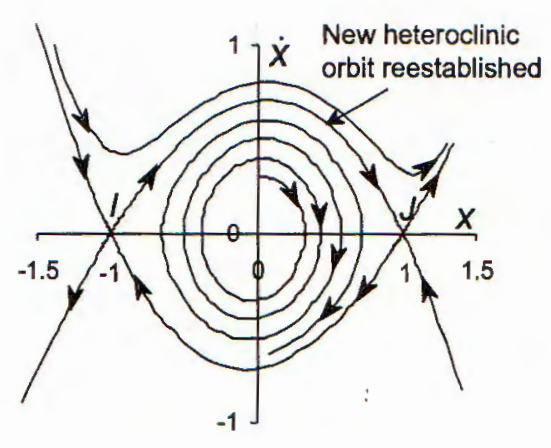

Fig. 6. The phase trajectories in the case $\beta=1, h=0.1, \alpha_{*}=4.992498$

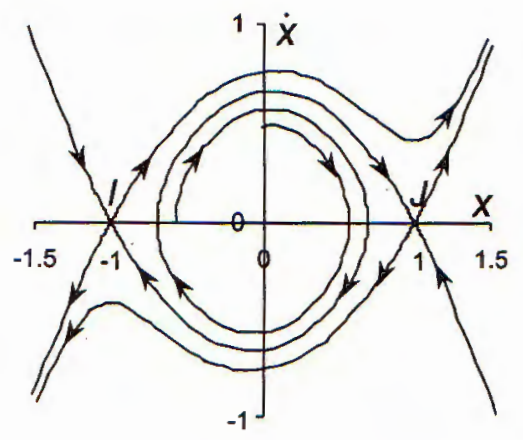

Fig. 5. The phase trajectories in $\beta=1, h=0.1, \alpha=1$

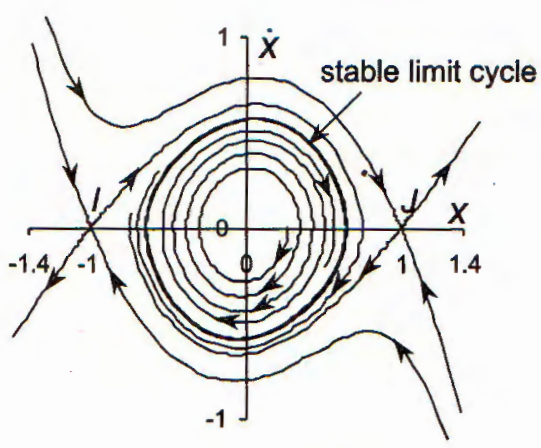

Fig. 7. The phase trajectories in the case $\beta=1, h=0.1, \alpha=10$

\section{VAN DER POL SYSTEMS WITH LINEAR PUSHING AND CUBIC ATTRACTING ELEMENTS}

The case of Van der Pol systems with linear pushing and cubic attracting elements is more complicated; it corresponds to the differential equation of the form

$$
\ddot{x}-x=-\beta x^{3}+h\left(1-\alpha x^{2}\right) \dot{x} ; \beta>0 .
$$

In the phase plane of the "original" system $(h=0)$, there are:

- Three critical points: a saddle at the origin $O$ and two stable centers at $I\left(-\frac{1}{\sqrt{\beta}}, 0\right)$ and $J\left(\frac{1}{\sqrt{\beta}, 0)}\right.$.

- Two homoclinic orbits, homoclinic to $O$, the left(right) encircles $I(J)$ and intersects the axis $x$ at $I^{\prime}\left(-\sqrt{\frac{2}{\beta}}, 0\right), J^{\prime}\left(\sqrt{\frac{2}{\beta}}, 0\right)$ respectively.

- Two types of free oscillations: the first with closed orbits lying inside each homoclinic orbit; the second with closed orbits lying outside both two homoclinic orbits.

Fig. 8 is drawn for $\beta=1, h=0$. 
As in the section 3 , the self - excitation $h\left(1-\alpha x^{2}\right) \dot{x}$ destroys both two homoclinic orbits to create four invariant manifolds except for a particular value $\alpha=\alpha_{*}$ for which two new homoclinic orbits appear .

For $\alpha \leq 1$, there is a unique closed orbit encircling all three critical points (the saddle $O$ and two unstable foci $I$ and $J$ ). This orbit represents a stable stationary self-excited oscillation (Figs. 9, 10).

For $1<\alpha<\alpha_{*}$, two new (left and right) closed orbits appear, respectively encircling $I, J$ which become now two stable foci. These two orbits correspond to unstable stationary self-excited oscillations (Fig. 11).

For $\alpha=\alpha_{*}$, two mentioned unstable closed orbits are connected at $O$ to create two new homoclinic orbits (Fig. 12).

For $\alpha>\alpha_{*}$, these two new homoclinic orbits are replaced by a big unstable closed orbit (Fig. 13).

Further, increasing $\alpha$, the two big orbits approach each other then disappear simultaneously. Figs. $8-13$ are drawn with the same values $\beta=1, h=0.8$, and correspond to $\alpha=0.7 ; 1 ; 1.15 ; \alpha_{*}=1.22947985 ; 1.25$.

As before, the Melnikov function can be used to determine the critical value $\alpha_{*}$. We also choose $\beta=1$. The Hamiltonian of the unperturbed system $(h=0)$ is $H(x, \dot{x})=\frac{x^{4}}{4}-$ $\frac{x^{2}}{2}+\frac{\dot{x}^{2}}{2}$ and the equation of the right homoclinic orbit is $H(x, \dot{x})=0$ or $\dot{x}= \pm \sqrt{x^{2}-\frac{x^{4}}{2}}$, here $(+)$ corresponds to the upper half orbit; $(-)$ corresponds to the lower one. The motion law along the homoclinic orbit satisfying the initial conditions $x(0)=\sqrt{2}, \dot{x}(0)=0$ is $x=\sqrt{2} \sec h(t)$. Hence, the value of the Menikov function can be easily calculated

$$
M=\int_{-\infty}^{+\infty} h\left(1-\alpha x^{2}(t)\right) \dot{x}^{2}(t) d t=\frac{4 h}{15}(5-4 \alpha) .
$$

The same result can be obtained without using the motion law

$$
\begin{aligned}
M & =2 h \int_{-\infty}^{0}\left(1-\alpha x^{2}(t)\right) \dot{x}^{2}(t) d t \\
& =2 h \int_{-\infty}^{0}\left(1-\alpha x^{2}(t)\right) \dot{x}(t) \cdot \dot{x}(t) d t \\
& =2 h \int_{0}^{\sqrt{2}}\left(1-\alpha x^{2}\right) \dot{x} d x=2 h \int_{0}^{\sqrt{2}}\left(1-\alpha x^{2}\right) \sqrt{x^{2}-\frac{x^{4}}{2}} d x \\
& =\frac{4 h}{15}(5-4 \alpha) .
\end{aligned}
$$

The critical value is thus equal to $\alpha=5 / 4=1,25$. A good agreement is observed for $\beta=1, h=0.1, \alpha_{*} \approx 1.249658$ found by the numerical method. 


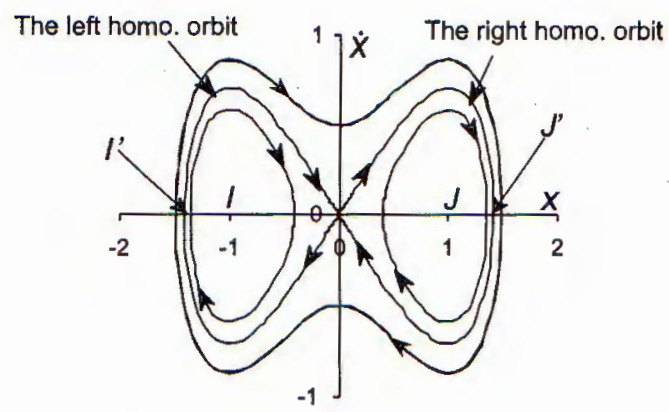

Fig. 8. The phase trajectories in the case

$$
\beta=1, h=0
$$

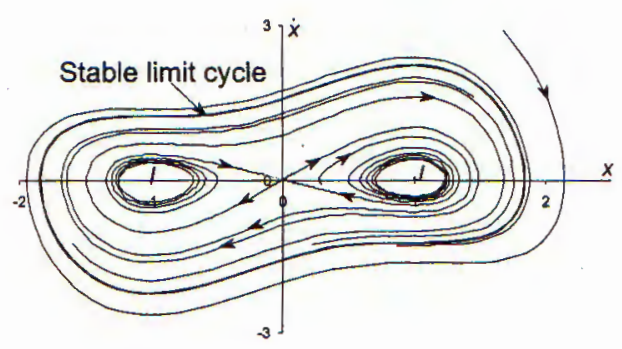

Fig. 10. The phase trajectories in the case $\beta=1, h=0, \alpha=1$

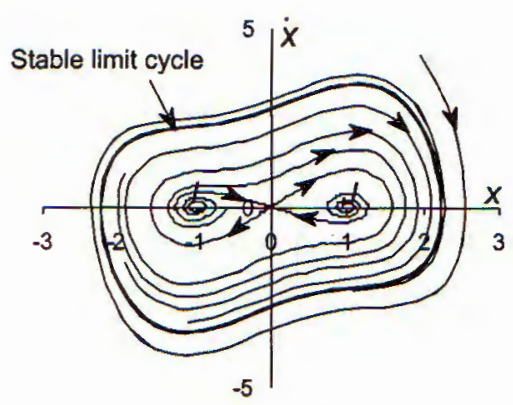

Fig. 9. The phase trajectories in the case $\beta=1, h=0.8, \alpha=0.7$

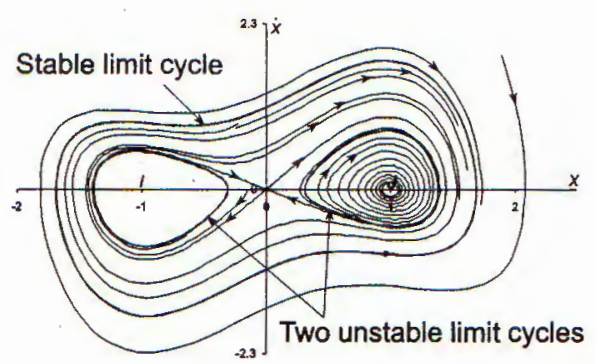

Fig. 11. The phase trajectories in the case $\beta=1, h=0.8, \alpha=1.15$

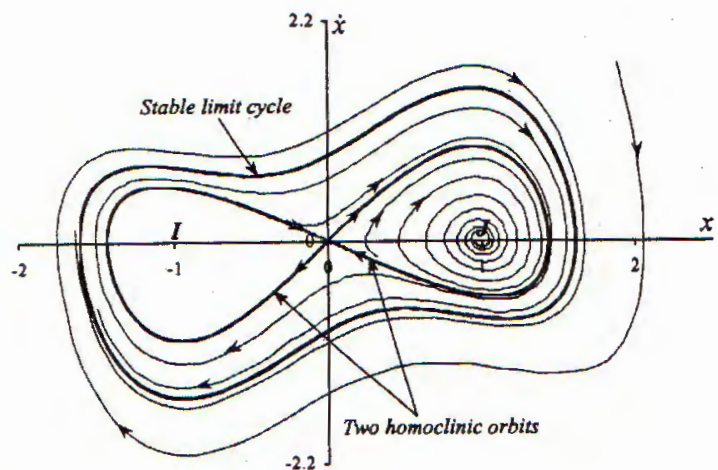

Fig. 12. The phase trajectories in the case $\beta=1, h=0.8, \alpha=1.22947985$

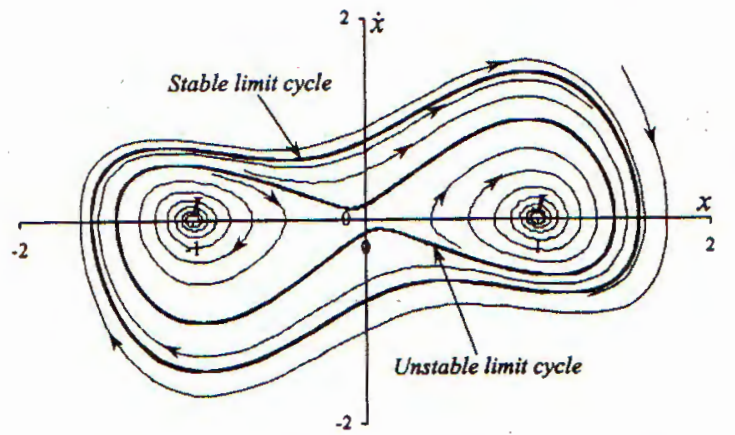

Fig. 13. The phase trajectories in the case $\beta=1, h=0.8, \alpha=1.25$

\section{CONCLUSION}

Van der Pol systems with various linear and cubic restoring elements are examined. It is shown that the evolution of stationary self - exited oscillations (closed orbits in the phase plane) depends mainly on the characters of restoring elements and on the positive element of self-excitation. 
This publication is completed with the financial support from the Council for Natural Science of Vietnam.

\section{REFERENCES}

1. I. G. Malkin, Some Problems of the Theory of Nonlinear Oscillations, Moscow, 1956 (in Russian).

2. Yu. A. Mitropolski, Nguyen Van Dao, Lectures on Asymptotic Methods of Nonlinear Dynamics, Vietnam National University Publishing House, 2003.

3. A. H. Nayfeh, B. Balachandran, Applied Nonlinear Dynamics, John Wiley and Son, Inc. New York, 1995.

4. Francis C Moon, Chaotic Vibration, John Wiley, 1996.

5. Steven H. Strogatz, Nonlinear Dynamics and Chaos, Westview Press, 2000.

Received October 12, 2005

Revised February 25, 2006

\section{CHẤN TƯ VAN ĐE PÔN VỚI CÁC DẠNG KHÁC NHAU CƯA YẾU TỐ PHỤC HỒI}

Một số chấn tử Van Đe Pôn với các dạng của yếu tố phục hồi bậc nhất và bậc ba được khảo sát. Đã chỉ ra quá trình tiến triển của chế độ dừng tự kích và vai trò của tự kích và của các yếu tố phục hồi. Phương pháp số trên máy tính được sừ dụng. 